\title{
20C, a novel bibenzyl compound, protected mice from MPTP/p injuries by regulating a-synuclein related inflammatory responds
}

\author{
Yu-He Yuan, Nai-Hong Chen \\ Institute of Materia Medica \& Neuroscience Center, Chinese Academy of Medical Sciences and Peking Union Medical \\ College, China
}

The novel bibenzyl compound 20C from Gastrodia elata plays a neuroprotective role in vitro, but its effects in vivo is unknown. In this study, we studied the effects of $20 \mathrm{C}$ and its associated-mechanisms on these effects on 1-methyl-4phenyl -1, 2, 3, 6-tetrahydropyridine/probenecide (MPTP/p) mouse model. Experiment results showed that the neuroprotective effects of 20C were as follows: improving behavioral deficit; attenuating DA depletion; reducing dopaminergic neuron loss; protecting BBB structure; ameliorating a-synuclein dysfunction, suppressing glial activation and regulating both the NF-kB signaling and the NLRP3 inflammasome pathways. All these results suggested that $20 \mathrm{C}$ might prevent the neurodegeneration of chronic MPTP/p treated mouse through regulating the a-synuclein related inflammatory responds, including BBB damages, glial activation, NF-kB signaling and NLRP3 inflammasome pathway.

*Correspondence to:

Yu-He Yuan, Department of Pharmacology, Institute of Materia Medica, Chinese Academy of Medical Sciences and Peking Union Medical College, Xiannongtan Street, Xicheng District, Beijing 100050, P.R. China, Tel: +86 1063165182 , Fax: +86 10 63165177, E-mail: yuanyuhe@imm.ac.cn

Nai-Hong Chen, Department of Pharmacology, Institute of Materia Medica, Chinese Academy of Medical Sciences and Peking Union Medical College, Xiannongtan Street, Xicheng District, Beijing 100050, P.R. China, Tel: +86 1063165177 , Fax: +86 10 63165177, E-mail: chennh@imm.ac.cn 